\title{
THE HÖRMANDER AND MASLOV CLASSES AND FOMENKO'S CONJECTURE
}

\author{
Z. TEVDORADZE
}

\begin{abstract}
Some functorial properties are studied for the Hörmander classes defined for symplectic bundles. The behaviour of the Chern first form on a Lagrangian submanifold in an almost Hermitian manifold is also studied, and Fomenko's conjecture about the behaviour of a Maslov class on minimal Lagrangian submanifolds is considered.
\end{abstract}

\section{INTRODUCTION}

In this work we are interestied in the characterustic classes of sympletic bundles and Lagrangian subbundles. One of such classes was discovered when studying asymptotic solutions of linear partial differential equations [1] and called a Maslov class. In [2] Arnold gives a pure geometric interpretation of the Maslov class.

A generalization of the Maslov class to higher cohomological dimensions was defined by Arnold and studied in [3]. Another generalization of the above-mentioned classes is defined by Trofimov in [4].

In [5] and [6] Hörmander defined the cohomology classes $\sigma(E ; X, Y) \in H^{1}(M ; \mathbb{Z})$ for arbitrary sections $X$ and $Y$ of Lagrange's Grassmanian $L(E) \stackrel{\pi}{\rightarrow} M$, where $E \stackrel{p}{\rightarrow} M$ is a sympletic fibre bundle. These classes are discribed in [7].

In [8] Fomenko formulates a conjecture that all Maslov-Arnold characteristic classes of minimal Lagrangian surfaces are equal to zero, and this conjecture is proved when Lagrangian surfaces are submanifolds in $M^{2 n}=\mathbb{R}^{2 n}=\mathbb{C}^{n}$.

The paper is organized as follows. In $\S 1$ we will describe the condition when the Maslov index $\ell(\gamma)$ is an even number for an arbitrary closed curve $\gamma$ in the Lagrangian manifold (Theorem 1.1) and prove one functorial property for Hörmander classes $\sigma(E ; X, Y)$ (Theorem 1.3). In $\S 2$ a class of Lagrangian manifolds $L H(M)$ is considered in an almost Hermitian manifold $M$ with the following property: $J^{*} \alpha_{H}$ is an exact 1-form in each manifold from $L H(M)$, where $J$ is an almost complex structure and $H$ is a mean curvature with respect to the inclusion in $M$. It is proved that $\left.c\right|_{N}$ is an exact 2-form for the Lagrangian manifold $N$ in the Hermitian manifold $M$ and $\left.c\right|_{N}=0$, if $N \in L H(M)$, where $c$ is the first Chern form. The extended variant of Fomenko's conjecture on the class $L H(M)$ also formulated and studied. This conjecture is proved for the class $L H\left(T^{*} Q^{n}\right)$, where $Q^{n}$ satisfies some additional condition (Theorem 2.8).

In conclusion we note that it remains unknown whether Fomenko's conjecture is true or not in the general case and how much the class $L H(M)$ extends the class of minimal Lagrangian submanifolds in $M$.

1991 Mathematics Subject Classification. 58F05, 57R20.

Key words and phrases. Symplectic manifold, connection, Maslov class, minimal surface, Hermitian manifold. 


\section{Construction of Maslov and Hörmander Classes}

Let $\left(V^{2 n}, \omega\right)$ be a symplectic vector space over $\mathbb{R}$. A real $n$-plane $\zeta$ in $V^{2 n}$ is called a Lgrangian plane if the restriction of $\omega$ on $\zeta$ vanishes. By $L(V)$ we denote Lagrange's Grassmanian which consists of all Lagrangian $n$-planes. It is well known that if we have a fixed point $X \in L(V)$, then $L(V)$ can be represented as a homogeneous space $U(n) / O(n)$, where the orthogonal group $O(n)$ is cannonically imbedded in the unitary group $U(n)$.

Let us consider the fibre bundle

$$
S U(n) / S O(n) \rightarrow U(n) / O(n) \stackrel{\operatorname{det}^{2}}{\rightarrow} S^{1},
$$

where the map $\operatorname{det}^{2}$ is a fibre map and $S U(n) / S O(n)$ is a fibre at the point $1 \in S^{1} \subset$ $\mathbb{C}$. The space $S U(n) / S O(n)$ is simply connected; therefore the long exact homotopy sequence of fibre bundles implies that $\pi_{1}(L(V))=\mathbb{Z}$ and hence $H_{1}(L(V) ; \mathbb{Z})=\mathbb{Z}$. From the formula of universal coefficients

$$
0 \rightarrow \operatorname{Ext}\left(H_{0}(L(V), \mathbb{Z})\right) \rightarrow H^{1}(L(V) ; \mathbb{Z}) \stackrel{h}{\rightarrow} \operatorname{Hom}\left(H_{1}(L(V) ; \mathbb{Z}) ; \mathbb{Z}\right) \rightarrow 0
$$

(where $(h\{f\})\left\{\sum c_{i} \otimes g_{i}\right\}=\sum f\left(c_{i}\right) \otimes g_{i},\{f\} \in H^{1}(L(V) ; \mathbb{Z}),\left\{\sum c_{i} \otimes g_{i}\right\} \in$ $\left.H_{1}(L(V) ; \mathbb{Z})\right)$, we obtain $H^{1}(L(V) ; \mathbb{Z})=0$.

The differential 1-form $\left(\operatorname{det}^{2}\right)^{*} \frac{d z}{2 \pi i z}$ is the generator of $H^{1}(L(V) ; \mathbb{Z})$, where the form $\frac{d z}{2 \pi i z}$ is the 1 -firm on $S^{1}=\{z \in \mathbb{C}:|z|=1\} \subset \mathbb{C}$.

Definition 1.1. A class od cohomology which is defined by 1-form $\left(\operatorname{det}^{2}\right)^{*} \frac{d z}{2 \pi i z}$ is called a Maslov class.

Definition 1.2. A submanifold $N \hookrightarrow V^{2 n}$ is called a Lagrangian manifold if the tangent plane at each point $x \in N$ is a maximal isotropic plane with respect to $\omega_{x}=\left.\omega\right|_{T_{x} V^{2 n}}$, i.e., $\left.\omega_{x}\right|_{T_{x} N}=0$.

For each closed curve $\gamma$ in $N$ there is a map

$$
G: \gamma \rightarrow L(V), \quad G(x)=\left(x, T_{x} N\right), \quad x \in \gamma,
$$

which is called Gaussian map. This map defines the integer

$$
\ell(\gamma)=\int_{\gamma}\left(\operatorname{det}^{2} \circ G\right)^{*} \frac{d z}{2 \pi i z}
$$

and thereby the class of cohomology from $H^{1}(N ; \mathbb{Z})$. This class does not depend on a choice of a Lagrangian plane $X \in L(V)$ and is called the Maslov class of a Lagrangian manifold $N$.

Theorem 1.3. If Gaussian map $G: \gamma \rightarrow L(V)$ can be covered by the continuous map $\widetilde{G}: \gamma \rightarrow U(n)$, i.e., $p \circ \widetilde{G}=G$, where $p: U(n) \rightarrow L(V)$ is the natural projection, then $\ell(\gamma)$ is an even number.

Proof. First notice that the map det $: U(n) \rightarrow S^{1}$ induces an isomorphism between the fundamental groups $\pi_{1}(U(n))$ and $\pi_{1}\left(S^{1}\right)$. Indeed, the long exact homotopy sequence of fibre bundles

$$
S U(n) \stackrel{j}{\rightarrow} U(n) \stackrel{\operatorname{det}}{\rightarrow} S^{1}
$$

contains the sequence

$$
\pi_{1}(S U(n)) \stackrel{j_{*}}{\rightarrow} \pi_{1}(U(n)) \stackrel{(\text { det })_{*}}{\rightarrow} \pi_{1}\left(S^{1}\right) \stackrel{\partial}{\rightarrow} \pi_{0}(S U(n)) .
$$


The map (det) ${ }_{*}$ is an isomorphism, since $S U(n)$ is a simply connected space.

It is clear that the map

$$
\pi_{1}\left(S^{1}\right) \ni[\gamma] \rightarrow \frac{1}{2 \pi i} \int_{\gamma} \frac{d z}{z} \in \mathbb{Z}
$$

is an isomorphism. The composition of (det) * and the above-mentioned map give the natural isomorphism

$$
\pi_{1}(U(n)) \ni[\gamma] \longmapsto \frac{1}{2 \pi i} \int_{\gamma} \frac{d(\operatorname{det} U)}{\operatorname{det} U} \in \mathbb{Z} .
$$

The projection $p: U(n) \rightarrow U(n) / O(n)$ defines the monomorpohism

$$
p_{*}: \pi_{1}(U(n)) \rightarrow \pi_{1}(L(V)),
$$

which is multiplication by 2 . This statement immediately follows from the commutative diagram

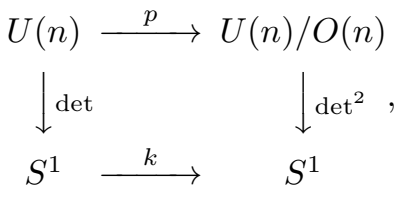

where $k(z)=z^{2}, z \in S^{1} \subset \mathbb{C}$. This monomorphism explicitly can be written as

$$
\mathbb{Z} \ni \frac{1}{2 \pi i} \int_{\gamma} \frac{d(\operatorname{det} U)}{\operatorname{det} U} \stackrel{p_{*}}{\longmapsto} \frac{1}{2 \pi i} \int_{\gamma} \frac{d\left(\operatorname{det}^{2} U\right)}{\operatorname{det}^{2} U} \in \mathbb{Z},
$$

where $\gamma$ is the closed curve is $U(n)$.

Now from the commutative diagram $p \circ \widetilde{G}=G$ we have $\ell(\gamma)=[G(\gamma)]=p_{*}[\widetilde{G}(\gamma)]$, where $[G(\gamma)] \in \pi_{1}(L(V))$ and $[\widetilde{G}(\gamma)] \in \pi_{1}(U(n))$. Clearly,

$$
\frac{\ell(\gamma)}{2}=\frac{1}{2 \pi i} \int_{\widetilde{G}(\gamma)} \frac{d(\operatorname{det} U)}{\operatorname{det} U} \in \mathbb{Z}
$$

Remark 1.4. The experience accumulated in investigating various concrete mechanical systems and calculations of the Maslov class for the Liouville tori of such systems show that an overwhelming majority of systems have got even numbers as components of the Maslov class [9], [10]. Theorem 1.3 describes the mechanism of such occurrence.

Now we briefly recall the definition and construction of more general classes, namely, of Hörmander classes [7].

Let $X, Y, Z, W$ be four Lagrangian $n$-planes in $V^{2 n}$ such that $Z \cap X=Z \cap Y=$ $\{0\}$ and $W \cap X=W \cap Y=\{0\}$. For them Hörmander defined the index at the intersection

$$
(X, Y, Z, W)=\frac{1}{2}\left(\operatorname{sign} Q_{Z}-\operatorname{sign} Q_{Z}\right)=\operatorname{ind} Q_{W}-\operatorname{ind} Q_{W},
$$

where $Q_{Z}$ and $Q_{W}$ are nondegenerate quadratic forms on $Y / X \cap Y$ defined by the formulas

$$
Q_{Z}(y)=\omega\left(p_{Z}^{X} y, y\right), \quad Q_{W}(y)=\omega\left(p_{W}^{X}(y), y\right)
$$


(here $p_{Z}^{X}$ and $p_{W}^{X}$ are the projections of $Y / Y \cap X$ onto $Z$ and $W$, respectively, accross $X)$. The following relations immediately follow from (1.3):

$$
\begin{aligned}
& (X, Y, Z, W)=-(X, Y, W, Z), \\
& (X, Y, Z, W)+(X, Y, W, V)+(X, Y, V, Z)=0, \\
& (X, Y, Z, W)=-(Z, W, X, Y) .
\end{aligned}
$$

Let $E \stackrel{p}{\rightarrow} M$ be a symplectic vector fibre bundle, i.e., for each point $m \in M$ there is a symplectic 2-form $\omega_{m}$ on $E_{m}$ which smoothly depends on $m$. Then we can consider the fibre bundle $L(E) \stackrel{\pi}{\rightarrow} M$, where $L(E)_{m}=L\left(E_{m}\right)$. Now let $X$ and $Y$ be the sections of the bundle $L(E)$, and $U=\left\{U_{\alpha}\right\}_{\alpha \in I}$ be an open covering of $M$ such that all nonempty finite intersections are diffeomorphic to $\mathbb{R}^{n}$ (such a covering is called a good covering). Then for arbitrary neighbouhoods $U_{\alpha}$ and $U_{\beta}$ one can find sections $Z_{\alpha}$ and $Z_{\beta}$ of $L(E)$ on $U_{\alpha}$ and $U_{\beta}$, respectively, such that $Z_{\alpha}(m)$ and $Z_{\beta}(m)$ are transversal to $X(m)$ and $Y(m)$ for each point $m \in U_{\alpha} \cap U_{\beta}$. In this situation the Cech 1-cocycle $\sigma$ can be defined by the formula

$$
\sigma\left(U_{\alpha}, U_{\beta}\right)=\left(X, Y, Z_{\alpha}, Z_{\beta}\right)
$$

The cohomology class $[\sigma]$ is called the Hörmander class for a symplectic vector fibre bundle $E$ and sections $X, Y$ and denoted by $\sigma(E ; X, Y) \in \check{H}^{1}(M ; \mathbb{Z})$.

From (1.4) and (1.5) one can conclude that

$$
\sigma(E ; X, Y)=-\sigma(E ; Y, X) .
$$

Let $\left(E^{\prime}, p^{\prime}, L(E)\right)$ be a pullback bundle of the bundle $E$ to $L(E)$ :

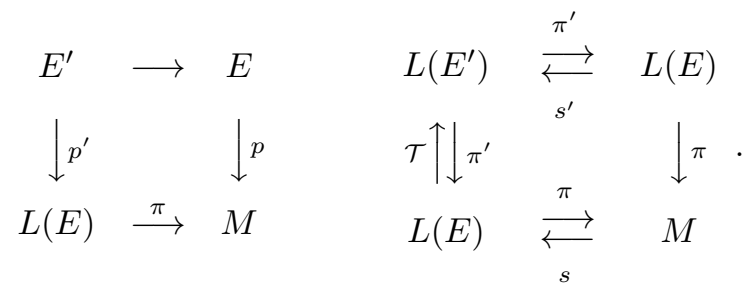

Then we can consider the fibre bundle $L(E) \stackrel{\pi^{\prime}}{\rightarrow} L(E)$ and the natural section $\mathcal{T}: \mathcal{L}(\mathcal{E}) \rightarrow \mathcal{L}\left(\mathcal{E}^{\prime}\right)$ which is defined by the formula $\mathcal{T}(\S)=\S$, where $x \in L(E)_{m}$ and the fibres $E_{x}^{\prime}, E_{m}$ are identified. An arbitrary section $S: M \rightarrow L(E)$ can be lifted to the section $S^{\prime}: L(E) \rightarrow L\left(E^{\prime}\right)$ by the formula $S^{\prime}(x)=S(m)$, where $x \in L(E)_{m}$, $m \in M$, i.e., if $x=(m, \xi)$ and $S(m)=(m, \eta)$, then $S^{\prime}(x)=(m, \xi, \eta)$ (here, as before, $L\left(E^{\prime}\right)_{x}$ and $L(E)_{m}$ are identified).

One can verify that $Y^{*}\left(S^{\prime}\right)=S$ and $Y^{*}(\mathcal{T})=\mathcal{Y}$ for each section $Y$ of the bundle $L(E)$.

It is not difficult to see that class $[\sigma]$ has got the following functorial property: if $\left(f^{*} E, f^{*}(p), M_{1}\right)$ is a pullback bundle of the bundle $(E, p, M)$, where $f: M_{1} \rightarrow M$ is a smooth map, then

$$
f^{*} \sigma(E ; X, Y)=\sigma\left(f^{*} E ; f^{*} X, f^{*} Y\right)
$$

$\left(f^{*} X, f^{*} Y\right.$ are pullback sections of the sections $X$ and $Y$ by the map $\left.f\right)$. 
The following equalities arise from (1.4) and (1.6):

$$
\begin{gathered}
\sigma\left(L\left(E^{\prime}\right) ; X^{\prime}, \mathcal{T}\right)-\sigma\left(\mathcal{L}\left(\mathcal{E}^{\prime}\right) ; \mathcal{Y}^{\prime}, \mathcal{T}\right)= \\
=\sigma\left(L\left(E^{\prime}\right) ; X^{\prime}, Y^{\prime}\right) \in \check{H}^{1}(L(E) ; \mathbb{Z}), \\
\sigma(E ; X, Y)=Y^{*} \sigma\left(L\left(E^{\prime}\right) ; X^{\prime}, \mathcal{T}\right) .
\end{gathered}
$$

For each section $X$ of the bundle $L(E)$ there is a characteristic class $\sigma_{X}$ defined by the formula

$$
\sigma_{X} \equiv \sigma\left(L(E) ; X^{\prime}, \mathcal{T}\right) \in \check{H}^{1}(L(E) ; \mathbb{Z})
$$

such that

i) the restriction of $\sigma_{X}$ on a fibre $L(E)_{m}, m \in M$, is the generator of $\check{H}^{1}\left(L(E)_{m} ; \mathbb{Z}\right)$;

ii) $X^{*} \sigma_{X}=0$.

The property ii) immediately follows from (1.8) and (1.6). Indeed, $X^{*} \sigma_{X}=X^{*} \sigma\left(L\left(E^{\prime}\right) ; X^{\prime}, \mathcal{T}\right)=\sigma\left(\mathcal{E} ; \mathcal{X}^{*}\left(\mathcal{X}^{\prime}\right), \mathcal{X}^{*}(\mathcal{T})\right)=\sigma(\mathcal{E} ; \mathcal{X}, \mathcal{X})=$ เ. The first property is more difficult and can be found in [7].

To prove Theorem 1.6 we will use the following well-known theorem [11].

Theorem 1.5 (Dold-Thom-Gysin). Let $h^{*}$ be a multiplicative cohomology theory and let $F \stackrel{i}{\rightarrow} E \stackrel{\pi}{\rightarrow} B$ be an $h^{*}$-fibration. Suppose there are elements $a_{1}, \ldots, a_{r}$ in $h^{*}(E)$ such that $\left(i^{*} a_{1}, \ldots, i^{*} a_{r}\right)$ is an $h^{*}($ point $)$-base for $h^{*}(F)$ as an $h^{*}($ point $)$ module; then $\left(a_{1}, \ldots, a_{r}\right)$ is an $h^{*}(B)$-base for $h^{*}(B)$-base for $h^{*}(E)$ as an $h^{*}(B)$ module.

Theorem 1.6. If $X$ and $Y$ are sections of the bundle $L(E)$, and $X^{\prime}, Y^{\prime}$ are the lifted sections of the bundle $L\left(E^{\prime}\right)$, then

$$
\sigma\left(L(E) ; X^{\prime}, Y^{\prime}\right)=\pi^{*} \sigma(E ; X, Y) .
$$

Proof. By virtue of the properties (i) and (ii) of the Hörmander class $\sigma_{X}$ and Theorem 1.5 we can write

$$
\sigma_{Y}=\pi^{*}[\beta(X, Y)]+k(X, Y) \sigma_{X}
$$

where $\beta(X, Y)$ is the closed 1-form on $M$ and $k(X, Y) \in \mathbb{Z}$. Applying $X^{*}$ and $Y^{*}$ to both sides of (1.11) and taking into account (1.8) and (ii), we respectively have

$$
\begin{aligned}
& \sigma(E ; X, Y)=[\beta(X, Y)]+k(X, Y) \cdot 0=[\beta(X, Y)], \\
& Y^{*} \sigma_{Y}=[\beta(X, Y)]+k(X, Y) \sigma(E ; X, Y)=0 .
\end{aligned}
$$

Now from (1.6), (1.12), (1.13) we have obtain $k(X, Y)=1$, and formula (1.11) acquires the form

$$
\sigma_{Y}=\pi^{*}(E ; Y, X)+\sigma_{X} .
$$

It is clear that (1.14) and (1.10) are equivalent equalities (see (1.8)).

\section{Fomenko's Conjecture}

Let $M^{2 n}$ be an almost Hermitian manifold, i.e., there exist a tensor field $J$ and a Riemannian metric $g$ on $M$, where $J$ is an endomorphism of the tangent bundle $T M$ with the property $J^{2}=-i d$ and $g$ is an invariant with respect to $J$, i.e., $g(J X, J Y)=g(X, Y)$ for all vector fields $X, Y \in \mathcal{T}(\mathcal{M})$ on $M$. The fundamental 2 -form on $M$ is defined by the formula

$$
\Phi(X, Y)=g(X, J Y), \quad X, Y \in \mathcal{T}(\mathcal{M}) .
$$


When $\Phi$ is a closed 2-form, $M$ is called an almost Kaehlerian manifold and $\Phi$ is called an almost Kaehler form on $M$. In this case $M$ is a symplectic manifold with a symplectic 2 -form $\Phi$. The converse is also true: for every symplectic manifold $\left(M^{2 n}, \omega\right)$ there exists an almost Hermitian structure on $M$ such that the fundamental 2 -form of this structure coincides with the symplectic 2 -form $\omega$. Thus every symplectic manifold is an almost Kaehlerian manifold [12].

Now let $N^{n}$ be a Lagrangian submanifold in an almost Kaehlerian manifold $M^{2 n}$, i.e., $i^{*} \Phi=0$, where $i: N \rightarrow M$ is the imbedding map. By $V N$ we denote the normal fibre bundle on $N$ with respect to the metric $g$ on $M$ (i.e., $V_{x} N$ is a $n$-plane in $T_{x} M$, which is orthogonal to $\left.T_{x} N, x \in N\right)$, and by $\nabla$ we denote the Riemannian connection on $M$. We recall that the trace of the following morphism of the fibrations

$$
B: T N \oplus T N \rightarrow V N, \quad B(x, y)=\left(\nabla_{x} Y\right)^{V}
$$

(where $x, y \in T_{n} N, n \in N$, and $Y$ is the locally defined vector field on $M$ which extends the vector $y,\left.Y\right|_{N}$ is the section of $T N$, and $\left(\nabla_{x} Y\right)^{V}$ is the normal component of vector $\nabla_{x} Y$ ), which can also be considered as a section of the fibration $V N$, is called the mean curvative of the submanifold $N$ and denoted by $H$. More precisely, if $e_{1}, \ldots, e_{n}$ is an orthobasis of the plane $T_{n} N$ and $V$ is a locally defined vector field on $M$ whose restriction on $N$ is a section of $V N$ and $V(n)=v$, then the mean curvature $H$ is defined by the relation

$$
g(H(n), v)=-\sum_{i=1}^{n} g\left(\nabla_{e_{i}} V, e_{i}\right)
$$

We recall that $N \stackrel{i}{\hookrightarrow} M$ is called a locally minimal submanifold if the mean curvature $H$ is zero at every point of $N$. From (2.1) we can conclude that the diagram

is commutative. By $\alpha_{H}$ we denote a section of the fibration $A^{1}(V N)$ (the fibration of the exterior 1-forms on $V N$ ) defined as

$$
\alpha_{H}(n)(X)=g(H(n), X), \quad n \in N, \quad X \in V_{n} N
$$

Definition 2.1. We say that the Lagrangian manifold $N$ in $M$ is locally minimal from the cohomological point of view if the 1 -form $\beta=J^{*} \alpha_{H}$ defines the trivial class of cohomology $H^{1}(N ; \mathbb{R})$.

The class of the above-defined submanifolds is denoted by $L H(M)$.

In [13] Le Hong Van, relying on the calibrated geometry methods developed in the fundamental work [14], shows that there exists an 1-form $\psi$ on $L(T M)$ such that the Lagrangian submanifold $N$ in $M$ is locally minimal if and only if

$$
G^{*}(\psi)=0,
$$

where $G: N \rightarrow L(T M)$ is the Gaussian map defined as $G(x)=\left(x, T_{x} N\right) \in$ $L(T M)_{x}, x \in N$.

Now we will briefly describe the 1 -form $\psi$.

Let $T_{x}^{c}\left(M^{2 n}\right)$ be the complexification of the tangent space of $M$ at point $x$, $x \in M^{2 n}$. By $S_{x}^{c}$ we denote a complex subspace of $T_{x}^{c}\left(M^{2 n}\right)$ which contains all eigenvectors of the operator $J$ with the eigenvalue $i$. The unitary bases $R^{c}(x)=$ $\left\{\varepsilon_{1}(x), \ldots, \varepsilon_{n}(x)\right\}$ of the space $S_{n}^{c}(x)$ sonstruct the principal bundle $U(M)$ over $M$, 
with the strucrure group $U(n)$. By $\varepsilon_{\overline{1}}, \ldots, \varepsilon_{\bar{n}}$ we denote the complex conjugate vectors of the vectors $\varepsilon_{1}(x), \ldots, \varepsilon_{n}(x)$. Then the vectors

$$
\begin{aligned}
& e_{i}(x)=\frac{1}{\sqrt{2}}\left(\varepsilon_{i}(x)+\varepsilon_{\bar{i}}(x)\right), \quad i=\overline{1, n}, \\
& e_{\bar{i}}(x)=\frac{i}{\sqrt{2}}\left(\varepsilon_{i}(x)-\varepsilon_{\bar{i}}(x)\right)=J e_{i}, \quad i=\overline{1, n},
\end{aligned}
$$

make up families of orthonormal real vectors.

An infinitesimal connection on the principal bundle $U(M)$ is called an almost Hermitian connection on $M$.

Let $U=\left\{U_{\alpha}\right\}_{\alpha \in I}$ be the open covering of the manifold $M$, which is equipped with local sections $S_{\alpha}: U_{\alpha} \rightarrow U(M)$; then an almost Hermitian connection $\pi$ can be defined by the given 1 -forms $\pi_{\alpha}$ on $U_{\alpha}, \alpha \in I$, with values in the Lie algebra $u(n)$. We can express $\pi_{\alpha}$ in terms of the $\left(\pi_{j}^{i}\right)_{i, j=\overline{1, n}}$ matrix, where $\pi_{j}^{i}$ are the 1 -forms on $U_{\alpha}$ and $\pi_{j}^{i}+\bar{\pi}_{i}^{j}=0$. If we define $S_{\alpha}$ as $S_{\alpha}(x)=R_{\alpha}^{c}(x), x \in U_{\alpha}$, then there exist metrices $B_{\beta}^{\alpha}(x) \in U(n)$ such that

$$
\begin{gathered}
S_{\alpha}(x) B_{\beta}^{\alpha}(x)=S_{\beta}(x), \\
\pi_{\beta}=\left(B_{\beta}^{\alpha}\right)^{-1} \pi_{\alpha} B_{\beta}^{\alpha}+\left(B_{\beta}^{\alpha}\right)^{-1} d B_{\beta}^{\alpha}, \quad \alpha, \beta \in I, \quad x \in U_{\alpha} \cap U_{\beta} .
\end{gathered}
$$

Frames of the type $R^{c}(x)$ and $\bar{R}^{c}(x)=\left\{\varepsilon_{\overline{1}}(x), \ldots, \varepsilon_{\bar{n}}(x)\right\}$ construct the principal bundle $U^{\prime}(M)$ on $M$ with a structure group $U(n)$ which is a subgroup in $U(2 n)$ by means of the imbedding $A \rightarrow\left(\begin{array}{cc}A & 0 \\ 0 & \bar{A}\end{array}\right), A \in U(n)$. Then the above-mentioned Riemannian connection $\nabla$ with respect to the metric $g$ can be expressed on each open set $U_{\alpha}$ by the matrix of 1-forms

$$
\left(\begin{array}{cc}
\pi_{j}^{i} & \pi_{\bar{j}}^{i} \\
\pi_{j}^{\bar{i}} & \pi_{\bar{j}}^{\bar{i}}
\end{array}\right),
$$

where $\pi_{\bar{j}}^{\bar{i}}=\overline{\pi_{j}^{i}}, \pi_{j}^{\bar{i}}=\overline{\pi_{\bar{j}}^{i}}$ ( since $\nabla$ is a real connection) and $\pi_{j}^{\bar{i}}+\pi_{\bar{j}}^{i}=0$ (for $\nabla g=0$ ).

The matrices $\left(\pi_{j}^{i}\right)$ (on each $U_{\alpha}$ ) define an almost Hermitian connection which is called the first canonical connection for an almost Hermitian manifold $M$.

From the first structure equations of E. Cartan we have

$$
d \theta^{i}=\theta^{k} \wedge \pi_{k}^{i}+\theta^{\bar{k}} \wedge \pi_{k}^{i}
$$

where $\theta^{j}=\frac{1}{\sqrt{2}}\left(e_{j}\right)^{*}+i\left(J e_{j}\right)^{*}, j=\overline{1, n}, k=\overline{1, n}$. (2.4) is equivalent to

$$
d \theta^{i}=\theta^{k} \wedge \pi_{k}^{i}+\Gamma_{\bar{k} q}^{i} \theta^{\bar{k}} \wedge \theta^{q}+\frac{1}{2} T_{\bar{k}}^{i} \theta^{\bar{k}} \wedge \theta^{\bar{q}}, \quad i=\overline{1, n}, \quad k=\overline{1, n}, \quad q=\overline{1, n}
$$

where $\Gamma_{\bar{k} q}^{i}$ are the coefficients of the Riemannian connection with respect to frames from the space $U^{\prime}(M)$, and $T_{\bar{k} \bar{q}}^{i}=\Gamma_{\bar{k} \bar{q}}^{i}-\Gamma_{\bar{q} \bar{k}}^{i}$. Now we note that the forms $T_{\bar{k} \bar{q}}^{i} \theta^{\bar{k}} \wedge \theta^{\bar{q}}$, $i=\overline{1, n}$, construct the vector 2 -form on $U(M)$ which coincides with the torsion vector $T$ of an almost cemplex structure. By direct calculation (using the fact that $d \Phi=0)$ we can obtain $\Gamma_{\bar{k} q}^{i}=0$. So (2.4) finally has the following view

$$
d \theta^{i}=\theta^{k} \wedge \pi_{k}^{i}+\frac{1}{2} T_{\bar{k}}^{i} \theta^{\bar{k}} \wedge \theta^{\bar{q}}, \quad i=\overline{1, n}, \quad k=\overline{1, n}, \quad q=\overline{1, n} .
$$


By $L(T M)$ we denote Lagrange's Grassmanian associated with the symplectic fibration $T M \stackrel{p}{\rightarrow} M$. The map $q: U(M) \rightarrow L(T M)$, defined as $q\left(\left(\varepsilon_{1}, \ldots, \varepsilon_{n}\right)\right)=$ $e_{1} \wedge \cdots \wedge e_{n}$ (here the multivector $e_{1} \wedge \cdots \wedge e_{n}$ is identified with the Lagrange $n$-plane span $\left.\left\{e_{1}, \ldots, e_{n}\right\}\right)$ defines the principal bundle with the structure group $O(n)$. On the space $U(M)$ there exists an 1-form

$$
\bar{\psi}=-\left(\sum_{k=1}^{n} i \pi_{k}^{k}+2 \operatorname{Im}\left(\sum_{i, k=1}^{n} T_{i k}^{i} \theta^{\bar{k}}\right)\right),
$$

which can be expressed as a pullback form by the map $q$. Indeed, the 1 -form $\sum_{k} \pi_{k}^{k}$ vanishes on the fibres of $q: U(M) \rightarrow L(T M)$ and is invariant under the action of the group $O(n)$ on $U(M)$. Therefore the form $\sum_{k} i \pi_{k}^{k}$ can be pulled down on the $L(T M)$. It is easy to verify that

$$
\left.\sum_{i}\left(\varepsilon_{\bar{i}}\right\lrcorner T^{i}\right)=\sum_{i, k} T_{\bar{i} \bar{k}}^{i} \theta^{\bar{k}}-\sum_{i, k} T_{\bar{k}}^{i} \bar{i} \theta^{\bar{k}}=2 \sum_{i, k} T_{\bar{i}}^{i} \bar{k} \theta^{\bar{k}}
$$

and if $R_{\alpha}(x)=R_{\beta}(x) A_{\alpha}^{\beta}(x)$, where $A_{\alpha}^{\beta}(x)=\left(A_{j}^{i^{\prime}}\right)_{i^{\prime}, j=\overline{1, n}} \in O(n), \alpha, \beta \in I$, then

$$
\left.\left.\left.\left.\sum_{i}\left(\varepsilon_{\bar{i}}\right\lrcorner T^{i}\right)=\sum_{i, i^{\prime}} A_{i}^{i^{\prime}}\left(\varepsilon_{\bar{i}^{\prime}}\right\lrcorner T^{i}\right)=\sum_{i, i^{\prime}, p} A_{i}^{i^{\prime}}\left(\varepsilon_{\bar{i}^{\prime}}\right\lrcorner T^{p^{\prime}}\right) A_{p^{\prime}}^{i}=\sum_{i^{\prime}}\left(\varepsilon_{\bar{i}^{\prime}}\right\lrcorner T^{i^{\prime}}\right) .
$$

This means that the form $2 \operatorname{Im}\left(T_{\bar{i}}^{i} \theta^{\bar{k}}\right)$ can also be pulled down and so there exists a 1 -form $\psi$ on $L(T M)$ such that

$$
\bar{\psi}=q^{*} \psi
$$

When $M$ is a Hermitian manifold $(T=0)$, the 1 -form $\psi$ on $L(T M)$ has a simpler form [8]

$$
\psi=J d f+d \theta
$$

where $f$ and $\theta$ are the locally defined functions on $L(T M)$.

Lemma 2.2. The diagram is commutative:

where $i$ is the imbedding map, $G$ is the Gaussian map.

Proof. Let $x \in N, D$ be an open neighbourhood of $x$ in $N$ and $S_{D}$ be a local section $S_{D}: D \rightarrow U(M), S_{D}(x)=\left(\varepsilon_{1}(x), \ldots, \varepsilon_{n}(x)\right)$. Let $S_{D^{\prime}}$ be the extension of $S_{D}$ to the tubular neighbourhood $D^{\prime}$ of $D$ in $M$; then a locally $n$-form on $D^{\prime}$

$$
\varphi=\operatorname{Re}\left(S_{D^{\prime}}^{*}\left(\theta^{1} \wedge \theta^{2} \wedge \cdots \wedge \theta^{n}\right)\right),
$$

is a $n$-form of comass 1 on $D^{\prime}$ and $\left.\varphi\right|_{D} \equiv 1$. If $X \in V_{n} N, n \in N$, we have

$$
\begin{aligned}
(X\lrcorner d \varphi)\left(e_{1}, \ldots, e_{n}\right) & =\sum_{i=1}^{n}(-1)^{i} e_{i}\left(\varphi\left(X, e_{1}, \ldots, \widehat{e}_{i}, \ldots, e_{n}\right)\right)+ \\
& +X\left(\varphi\left(e_{1}, \ldots, e_{n}\right)\right)+ \\
& +\sum_{i<j}(-1)^{i+j} \varphi\left(\left[e_{i}, e_{j}\right], X, \ldots, \widehat{e}_{i}, \ldots, \widehat{e}_{j}, \ldots, e_{n}\right)+ \\
& +\sum_{i=1}^{n}(-1)^{i} \varphi\left(\left[X, e_{i}\right], e_{1}, \ldots, \widehat{e}_{i}, \ldots, e_{n}\right) .
\end{aligned}
$$


Since $\varphi$ has comass $1, X\left(\varphi\left(e_{1}, \ldots, e_{n}\right)\right)=0$, and $\left.X\right\lrcorner \varphi \equiv 0$ for all $X \perp T_{n} N$. Thus from the above equality we have

$$
\begin{aligned}
(X\lrcorner d \varphi)\left(e_{1}, \ldots, e_{n}\right) & =\sum_{i=1}^{n}(-1)^{i} \varphi\left(\left[X, e_{i}\right], e_{1}, \ldots, \widehat{e}_{i}, \ldots, e_{n}\right)= \\
& =-\sum_{i} g\left(\left[X, e_{i}\right], e_{i}\right)=-\sum_{i} g\left(\nabla_{X} e_{i}^{\prime}-\nabla_{e_{i}} X, e_{i}\right)= \\
& =\sum_{i} g\left(\nabla_{e_{i}} X, e_{i}\right)=-g(H, X) .
\end{aligned}
$$

In the fourth equality the fact is used that $\nabla_{X}\left\langle e_{i}^{\prime}, e_{i}^{\prime}\right\rangle=0$, and $e_{i}^{\prime}$ denotes the locally defined vector fields on $D^{\prime}$ which extend the vectors $e_{i}, i=\overline{1, n}$. By direct calculation we can obtain $\left.\left(S_{D}\right)^{*}(\bar{\psi})(J X)=(X\lrcorner d \varphi\right)\left(e_{1}, \ldots, e_{n}\right)=-\alpha_{H}(X)$. Since $q \circ S_{D}=G$, we have

$$
\alpha_{H}(X)=-G^{*} \psi(J X)=-J^{*} G^{*} \psi(X) .
$$

Therefore

$$
\beta=J^{*} \alpha_{H}=G^{*} \psi
$$

The second structure equations of E. Cartan for the first canonical connection $\pi=\left(\pi_{j}^{i}\right)_{i, j=\overline{1, n}}$ have the form

$$
\Omega=d \pi+\pi \wedge \pi
$$

where $\Omega$ is the curvature form for the connection $\pi$. Taking into account $\pi_{j}^{i}=-\bar{\pi}_{i}^{j}$, from (2.10) we have $\sum_{k} \Omega_{k}^{k}=-\sum_{k} \bar{\Omega}_{k}^{k}$ and $d\left(\sum_{k} \pi_{k}^{k}\right)=\sum_{k} \Omega_{k}^{k}$. The form $\frac{i}{2 \pi} \sum_{k} \Omega_{k}^{k}$ is real and can be pulled down on $M$. It is called the Chern first form and denoted by $c$. By (2.6) we obtain

$$
d \bar{\psi}=-2 \pi p^{*} c-2 d\left(\operatorname{Im} \sum_{i, k} T_{\bar{i}}^{i} \bar{k} \theta^{\bar{k}}\right) .
$$

For an open covering $U=\left\{U_{\alpha}\right\}_{\alpha \in I}$ of the manifold $M$ there exists a commutative diagram

Since $p^{*} c=q^{*} \pi^{*} c$ and $G^{*} \pi^{*}=i^{*}$, we have

$$
S_{U_{\alpha}}^{*} d \bar{\psi}=-\left.2 \pi c\right|_{N_{\alpha}}-2 S_{U_{\alpha}}^{*} d\left(\operatorname{Im} \sum T_{\bar{i} \bar{k}}^{i} \theta^{\bar{k}}\right)
$$

Now, taking into account equality $(2.9)$ and $S_{U_{\alpha}}^{*} q^{*}=i_{\alpha}^{*} G^{*}$, we obtain

$$
i_{\alpha}^{*} d \beta=i_{\alpha}^{*} G^{*} d \psi=-\left.2 \pi c\right|_{N_{\alpha}}-2 d\left(S_{U_{\alpha}}^{*} \operatorname{Im}\left(\sum_{i, k} T_{i}^{i} \bar{k} \theta^{\bar{k}}\right)\right) .
$$

Thus $\beta$ is the global 1-form on $N$, the expression $d\left(S_{U_{\alpha}}^{*} \operatorname{Im}\left(\sum_{i, k} T_{\bar{i}}^{i} \bar{k} \theta^{\bar{k}}\right)\right.$ defines the global closed 2-form on the Lagrangian submanifold $N$ and we denote it by $\gamma$. We have 
Proposition 2.3. The Chern form $c$ on the Lagrangian submanifold $N$ in an almost Hermitian manifold $M$ can be expressed by the sum

$$
i^{*} c=-\frac{1}{2 \pi} d \beta-\frac{1}{\pi} \gamma,
$$

where $i$ is the imbedding map $i: N \rightarrow M$, the forms $\beta$ and $\gamma$ depend on the mean curvature and torsion vector form, respectively.

Theorem 2.4. If $M$ is a Hermitian manifold then:

i) the form $\pi^{*} c$ is an exact form on $L(T M)$;

ii) if $N$ is a Lagrangian submanifold in $M$ then $i^{*} c=-\frac{1}{2 \pi} d \beta$;

iii) the form $\psi$ is a closed form exactly when $c=0$;

vi) if $\beta$ is closed on $N$ (in particular, when $N \in L H(M)$ ) then $i^{*} c=0$;

$\mathrm{v})$ if $M$ is the Kaehlerian manifold then $\psi$ is closed exactly when Ricchi's tensor is identicaly equal to zero.

Proof. From formulas (2.11), (2.9) we have

$$
q^{*} d \psi=-2 \pi p^{*} c
$$

Taking into account $\pi \circ q=p$, we obtain

$$
q^{*}\left(d \psi+2 \pi \cdot \pi^{*} c\right)=0
$$

As $q: U(M) \rightarrow L(T M)$ is a fibration, the above equation implies

$$
\pi^{*} c=-\frac{1}{2 \pi} d \psi
$$

By formula (2.14) it is clear that $\psi$ is closed exactly when $c=0$. Now we recall that the form $c$ on the Kaehlerian manifold can be locally expressed by the formula (see [12])

$$
c=\frac{1}{2 \pi} \sum_{i, j} R_{i \bar{j}} d z^{i} \wedge d z^{\bar{j}} .
$$

Formulae (2.14), (2.15) prove assertions i), iii), v). (2.13) immediately implies ii) and iv).

When $M=\mathbb{C P}^{n}$ and $N \hookrightarrow \mathbb{C P}^{n}$ is Lagrangian manifold, the conditions of Theorem 2.4 are fulfilled for Chern forms of higher dimension on $\mathbb{C P}^{n}$. This result follows from the well-known fact that the Chern forms on $\mathbb{C P}^{n}$ are calculated by the formula

$$
c_{k}=m(k) c^{k}, \quad k=\overline{1, n}, \quad m(k) \in \mathbb{N} .
$$

Now lat $X$ and $Y$ be the sections of the bundle $i^{*} L(T M)$ on $N$, where $N$ is a submanifold in $M$. By Theorem 1.5, for every closed 1-form $\varphi$ on $L(T M)$ there exists a 1 -form $\delta$ on $N$ such that

$$
\widehat{i}^{*}[\varphi]=\pi_{1}^{*}[\delta]+k([\varphi], X) \sigma_{X}, \quad k([\varphi], X) \in \mathbb{R}
$$

here $\widehat{i}$ is an imbedding map $i^{*} L(T M) \stackrel{\widehat{i}}{\rightarrow} L(T M)$ and $\pi_{1}$ is the projection of the bundle $i^{*} L(T M)$. Applying $X^{*}$ and $Y^{*}$ to (2.16), we obtain respectively

$$
X^{*} \widehat{i}^{*}[\varphi]=[\delta] ; \quad Y^{*} \widehat{i}^{*}[\varphi]=[\delta]+k([\varphi], X) \sigma\left(i^{*}(T M) ; X, Y\right) .
$$

From the above equations we have

$$
k([\varphi], X) \sigma\left(i^{*}(T M) ; X, Y\right)=Y^{*} \widehat{i}^{*}[\varphi]-X^{*} \widehat{i}^{*}[\varphi]
$$


In the particular case, when $\widehat{i}^{*}[\varphi]$ is not a pullback class from $H^{1}(N ; \mathbb{R})$, we have $k \neq 0$ and therefore (2.18) can be rewritten as

$$
\sigma\left(i^{*}(T M) ; X, Y\right)=\frac{1}{k([\varphi], X)}\left(Y^{*} \widehat{i}^{*}[\varphi]-X^{*} \widehat{i}^{*}[\varphi]\right)
$$

Thus we have proved

Proposition 2.5. For an arbitrary closed 1-form $\varphi$ on $L(T M)$ (it is assumed that $\widehat{i}^{*}[\varphi]$ is not a pullback class from $H^{1}(N ; \mathbb{R})$ by the map $\left.\pi_{1}\right)$, the Hörmander class $\sigma\left(i^{*}(T M) ; X, Y\right)$ can be expressed by formula $(2.19)$.

Proposition 2.6. If $N$ is a manifold from $L H(M)$ and $\psi$ is the closed 1-form on $L(T M)$, then

$$
\widehat{i}^{*}[\psi]=k([\psi], G) \sigma_{G} .
$$

Proof. For the 1-form $\psi$ and the Gaussian section $G$ (2.16) can be rewritten as

$$
\widehat{i}^{*}[\psi]=\pi_{1}^{*}[\delta]+k([\psi] ; G) \sigma_{G} .
$$

Applying $G^{*}$ to the formula and taking into account $N \in L H(M)$ and Lemma 2.2, we conclude that $[\delta]=0$.

Now let $M^{2 n}=T^{*} Q^{n}$ be a cotangent bundle and $\omega$ be a canonical symplectic structure on $M^{2 n}$. $M^{2 n}$ can be considered as an almost Kaehlerian manifold with the fundamental 2 -form $\omega$. There is a fixed section $A$ of the fibre bundle $L\left(T M^{2 n}\right) \stackrel{\pi}{\rightarrow} M^{2 n}$, which is defined at every point $m \in M^{2 n}$ as a tangent plane at the point $m$ of the fibre $p^{-1}(p(m))$, where $p$ is a natural projection $T^{*} Q^{n} \stackrel{p}{\rightarrow} Q^{n}$.

For every Lagrangian submanifold $N^{n} \stackrel{i}{\hookrightarrow} M^{2 n}$, the Gaussian map $G: N^{n} \rightarrow$ $i^{*} L\left(T M^{2 n}\right)$ is defined as previously. The following definition is equivalent to Definition 1.1 .

Definition 2.7. The cohomology class $\sigma\left(i^{*}(T M) ; G, A\right) \in H^{1}(N ; \mathbb{Z})$ is called the Maslov class of the Lagrangian submanifold $N$ and we denote it by $\sigma_{N}$.

Theorem 2.8. Let $N^{n}$ be a manifold from $L H\left(M^{2 n}\right)$. Then:

i) if $H^{1}\left(Q^{n} ; \mathbb{R}\right)=0$ and $\psi$ is a closed 1-form, then $k([\psi] ; A) \sigma_{N}=0, k([\psi], A) \in$ $\mathbb{R}$;

ii) if $H^{1}\left(Q^{n} ; \mathbb{R}\right)=0$ and $[\psi] \neq 0$, then $\sigma_{N}=0$.

Proof. In condition i) we have $[\psi]=k([\psi] ; A) \sigma_{A}$ so that $k([\psi] ; A) \sigma_{N}=-G^{*} \widehat{i}^{*}[\psi]=$ $-[\beta]=0$.

If $[\psi] \neq 0$, then $k([\psi] ; A) \neq 0$ and ii) follows from i).

Remark 2.9. When $M=\mathbb{R}^{2 n}$, all conditions of Theorem 2.5 are satisfied. The form $\psi$ is a closed 1-form, more precisely, $\psi=d \theta$ (see formula 2.8), where $\theta$ is the function from $L\left(T \mathbb{R}^{2 n}\right)=\mathbb{R}^{2 n} \times U(n) / O(n)$ to $S^{1}$. It is not difficult to calculate that in this case $k([\psi] ; A)=2 \pi$. Therefore Theorem 3 in [8] (Fomenko's conjecture) follows from Theorem 2.8 as a corollary not only for minimal Lagrangian submanifolds in $\mathbb{R}^{2 n}$ as in [8], but already for the manifolds from $L H(M)$ as well.

By Remark 2.9 and Theorem 2.8 we are able to extend Fomanko's conjecture for the manifolds from the class $L H\left(M^{2 n}\right)$. When the form $\psi$ is a closed 1-form, this conjecture equivalently can be reduced to the exactness of the form $\left.A^{*}[\psi]\right|_{N}$. 
Corollary 2.10. If $M^{n}$ is a Kaehlerian manifold, with $H^{1}\left(M^{n} ; \mathbb{R}\right)=0$, and Ricchi's tensor is identically equal to zero, then for $N \in L H\left(T M^{n}\right)$ we have $k([\psi], A) \sigma_{N}=0$. In the particular case, where $[\psi] \neq 0$, we have $\sigma_{N}=0$.

This corollary follows from Theorems 2.4 and 2.8.

\section{AKNowledgement}

The research described in this paper was made possible in part by Grant No. BJV000 from the International Science Foundation.

\section{REFERENCES}

1. V. P. Maslov, Theory of perturbation and asymptotic methods. (Russian) Moscow University Press, Moscow, 1965.

2. V. I. Arnold, Characteristic class entering in quantization conditions. (Russian) Funkts. Anal. Prilozh. 1(1967), No. 1, 1-14.

3. D. B. Fuks, Maslov-Arnold characteristic classes. (Russian) Dokl. Akad. Nauk SSSR 178(1968), No. 2, 303-306.

4. V. V. Trofimov, Symplectic connections, index of Maslov and conjecture of Fomenko. (Russian) Dokl. Akad. Nauk SSSR 304(1989), No. 6, 1302-1305.

5. L. Hörmander, Fourier integral operators I. Acta Math. 127(1971), 79-183.

6. L. Hörmander, The calculus of Fourier integral operators. Prospects in Math., 33-57, Ann. of Math. Stud., 70, Princeton Univ. Press., Princeton, N. Y., 1971.

7. V. Guilleman and S. Sternberg, Geometric Asymptotics. Amer. Math. Soc., Providence, Rhode Island, 1977.

8. Le Hong Van and A. T. Fomenko, The criteria of minimality of Lagrangian submanifolds in a Kaehlerian manifold. (Russian) Mat. Zametki 4(1987), 559-571.

9. Z. A. Tevdoradze, One note on the calculation of Arnold-Maslov class of Liouville tori in cotangent bundles. (Russian) Uspekhi Mat. Nauk 43(1988), No. $5,219-220$.

10. Z. A. Tevdoradze, Calculation of Maslov class in the Lagrange and Kovalevskaya problem for the motion of a rigid body about a fixed point. (Russian) Bull. Acad. Sci. Georgia 141(1991), No. 1, 45-49.

11. E. Dyer, Cohomology theories. W. A. Benjamin Inc., New York, 1969.

12. A. Lichnerowicz, Théorie globale des connexions et des groups d'holonomie. Ed. Cremonese, Roma, 1955.

13. Le Hong Van, Minimal $\Phi$-Lagrangian surfaces in almost Hermitian manifolds. (Russian) Mat. Sb. 180(1989), 924-936.

14. R. Harvey and H. B. Lawson, Calibrated geometries. Acta Math. 148(1982), $47-157$.

Faculty of Mechanics and Mathematics I.Javakhishvili Tbilisi state University 2,University St., Tbilisi 380043, Republic of Georgia

E-mail address: tevzaz@ti.net.ge 